Horizons philosophiques

\title{
Frontières des études littéraires : science de la littérature, science des discours
}

\section{Marc Angenot}

Volume 1, numéro 1, automne 1990

Sémiotiques $1:$ mises au point, mises en question

URI : https://id.erudit.org/iderudit/800859ar

DOI : https://doi.org/10.7202/800859ar

Aller au sommaire du numéro

Éditeur(s)

Collège Édouard-Montpetit

ISSN

1181-9227 (imprimé)

1920-2954 (numérique)

Découvrir la revue

\section{Citer cet article}

Angenot, M. (1990). Frontières des études littéraires : science de la littérature, science des discours. Horizons philosophiques, 1(1), 23-34.

https://doi.org/10.7202/800859ar d'utilisation que vous pouvez consulter en ligne.

https://apropos.erudit.org/fr/usagers/politique-dutilisation/ 


\section{Frontières des études littéraires : science de la littérature, science des discours.}

Que la notion de littérature, avec grosso modo l'extension et la compréhension que nous lui accordons ne se soit dégagée peu à peu dans les cultures européennes qu'à la fin du XVIIIe puis au XVIIIe siècles et soit absolument désagrégée aujourd'hui est une évidence admise partout'. Que la notion grecque des "Muses", lesquelles comprennent l'histoire et l'astronomie dans un même ensemble avec ce qu'anachroniquement nous appellerions la "littérature", que cette notion relève d'une logique culturelle qui nous est fondamentalement étrangère, presque inintelligible, n'est pas moins évident. Que la Chine, le Japon, la Corée aient construit des catégories de discours sublimes, de haute légitimité sociale qui ne se superposent aux catégories occidentales des belles lettres et aux catégories modernes de littérature, de fiction, qu'au prix de «forçages" et d'abus de langage n'est pas moins certain - de même que la catégorie de "philosophie» ne s'applique à certaines traditions de l'Extrême-Orient que par une analogie discutable et trompeuse. Plus personne ne soutient des théories

1. Cet article provient d'une communication au colloque afrontières de la littératurew organisé par le département de littérature comparée de l'Université de Montréal le 25 mars 1988. 
qui feraient penser à celles de Shaftesbury ou de Kant selon quoi il existe une faculté spécifiquement esthétique chez l'homme qui, éduquée, forme le goût et à quoi répondent les caractères particuliers de l'œuvre d'art. Nul ne semble avoir entrepris de réfuter les thèses par lesquelles Tzvetan Todorov ouvre Les Genres du discours, à savoir qu'il est impossible de donner une définition structurelle tenable de la "littérature", de même qu'il est impossible de ramener à une axiomatique homogène l'ensemble des genres qu'un constat historique empirique regroupe à un moment donné dans l'ordre du “littéraire»?2.

Nous savons tous que l'objet desdites «études littéraires» est et demeurera évanescent. La «littérarité» des textes du canon des belles lettres propre à une société donnée ne saurait suffire à circonscrire un objet d'étude puisque tous les théoriciens qui ont repris la problématique de la literaturnost, ont fini par se convaincre :

1) qu'il y avait en abondance des traits de littérarité, et de la polysémie dans des énoncés et textes non littéraires, - dans des slogans politiques par exemple selon la discussion célèbre de R. Jakobson ${ }^{3}$;

2) de Mukarovsky à Genette et Fitch, que la «littérarité" d'un texte tient essentiellement à une attitude de réception, qu'on peut donc lire avec une attitude littéraire un fait divers du journal, qu'une certaine lecture métamorphosera ce fait divers en texte littéraire4;

3) que l'intérêt, le plaisir, la valeur que l'on peut percevoir dans un texte littéraire ne tiennent pas à leurs marques formelles de littérarité, mais à des effets pathétiques, à des projections psychologiques, à des conjectures exégétiques et à des manipulations cognitives, des schémas

2. Tzvetan Todorov, Les Genres du discours, Paris, Seuil, 1978, chap.l.

3. Roman Jakobson, "Linguistics and Poetics", dans T. Sebeok, Style in Language, Cambridge, MIT Press, 1960, p. 30-77.

4. Voir notamment Gérard Genette, Figures II, Paris, Seuil, 1969, p. 150. 
gnoséologiques qui se réalisent peut-être dans les textes dits littéraires de façon "curieuse", "étrange» et complexe, mais qui, une fois encore, ne leur sont pas propres.

Il est certes possible de donner une définition relative et historique de l'objet "littérature»: c'est alors ce qu'une société à un moment donné a "canonisé" sous ce nom. Cette production, qui intéresse certains sociologues du "champ littéraire», n'intéresse cependant guère les théoriciens et historiens littéraires; elle englobe empiriquement tout ce qui a été publié comme littérature avec quelque marque de légitimité (statut des éditeurs, réception, comptes rendus), mais justement cette masse, c'est ce dont les études littéraires ne veulent pas s'occuper : des 500 romans, des 800 recueils de vers, des 150 récits de voyages, etc., publiés dans le champ littéraire annuellement en France à la fin du XIXe siècle par exemple.

Le flou, l'indétermination radicale de l'objet rejaillit contradictoirement sur le champ d'application des méthodes "propres" aux études littéraires, - car ces méthodes ne sauraient leur être propres en aucun sens rigoureux. La Rhétorique d'Aristote a été pensée pour théoriser des formes de discours persuasifs que nous ne considérons pas "littéraires» (tout en ne sachant du reste pas trop si l'essai, la polémique, le pamphlet relèvent des études littéraires, pas plus que nous ne sommes prêts à dire que les schémas argumentatifs et les stratégies persuasives de la Rhétorique d'Aristote sont réellement utiles à quiconque étudie des "essais" modernes.) Le Traité des Tropes de Du Marsais travaille bien sûr avec des exemples que nous reconnaissons pour "littéraires", mais les tropes euxmêmes (dont Du Marsais disait qu'il s'en fait plus un jour de marché aux Halles, qu'au cours d'une séance à l'Académie) n'ont rien de propre au littéraire 5 . La métaphore, la métonymie, la synecdoque ne sont plus pour nous ni des

5. César Chesneau Du Marsais, Des Tropes, Paris, Barrois, $1781,1^{\circ}$ éd. 1730. 
"figures rhétoriques» ni des «figures littéraires", mais des mécanismes génétiques fondamentaux de toute semiosis. La narratoloqie, qui est un des secteurs où le consensus des critiques littéraires sur quelques notions fondamentales est le mieux établi, n'a rien encore de méthodologiquement spécifique. Elle nous vient d'un ethnographe, Vladimir Propp, travaillant sur des contes oraux qui ne sont littéraires que par abus de langage et anachronisme. Les meilleures études narratologiques sont - parmi d'autres celles de Greimas et de ses disciples du groupe d'Entrevernes travaillant sur les Évangiles, celles de Hayden White travaillant sur la narration historiographique ${ }^{6}$. Les littéraires, en possession d'un instrument assez riche pour justifier toutes sortes de formes du récit, sont partis aussitôt utiliser cette batterie de notions et de paradigmes, qui sur les "études de cas" de Freud or de Stekel, qui sur le fait divers du journal, qui sur Plutarque et qui sur Karl Marx. Ils avaient raison : il n'est si bel instrument qui ne se rouille à ne rien faire.

À ce point vous vous demandez : «Pourquoi nous ditil tout cela, nous le savons parfaitement, nous l'enseignons constamment et le confessons publiquement; il ne va pas continuer à accumuler toutes les preuves circonstancielles de l'inexistence d'une identité du fait littéraire, d'une homogénéité interne du corpus, d'une quelconque problématique spécifique et d'une méthodologie propre". Je crois en effet que nous le savons, mais j'ajoute que l'institution universitaire globalement le sait sur le mode du "Je ne veux pas le savoir."

Je ne veux pas le savoir, ou du moins je prétends n'en pas tirer les conséquences avec rigueur, dussé-je être accusé d'un peu glorieux raisonnement par les consé-

6. Voir Signes et paraboles par le groupe d'Entrevernes, postface d'A.-J. Greimas, Paris, Seuil, 1977 et Hayden White, The Content of Form. Narrative Discourse and Historical Representation, Baltimore et Londres, Johns Hopkins University Press, 1987. 
quences, fâcheuses pour moi et pour la prospérité de mes bases institutionnelles ! Ou bien, au contraire, vous allez me concéder tout ceci - pas d'objet, pas de critère discriminatoire, pas de méthodologie - mais m'opposer en fin de compte un argument d'évidence qui, par son évidence même, balaye mon argumentation selon laquelle il y a bien une différence entre un livre de cuisine et Kafka, entre un éditorial de journal et Musil, et donc que la littérature dans son inquiétante étrangeté existe sans qu'on en puisse offrir le concept positivement. Dans La Lettre et la Voix, Paul Zumthor redémontre avec force qu'il "n'existe pas de catégorie de "littérature" en soi ${ }^{7}$, que "la littérature" n'existe (n'existe encore) que comme partie d'un tout chronologiquement singulier, reconnaissable à diverses marques (telles l'existence de disciplines parasitaires dénommées "critique" ou "histoire" littéraires ${ }^{8}$ ), mais on peut lui répliquer par un argument ad hominem qu'il recevrait de bonne grâce, que c'est pourtant de littérature médiévale qu'il s'occupe, fût-ce en consacrant 350 pages à démontrer l'impossibilité de la connaître en lui appliquant nos catégories ou quelque catégorie spécifique que ce soit. Zumthor retrouve au bout du compte la thèse qui a fait les belles années de la période symboliste, qui est que le texte littéraire est "une activité ayant en soi sa propre fin", opposée ainsi à toute production discursive dont «le projet demeure inséparable (...) d'un projet global de société où [elle] s'intégre[rait] fonctionnellement»; que le "roman" médiéval et le "grand chant courtois" ont à voir avec ces «jeu, détour, ruse discursive» qui font qu'on peut en parler comme de la littérature ${ }^{9}$. Zumthor tient les deux bouts du dilemme : la littérature est indéfinissable, elle n'a pas de

7. Paul Zumthor, La lettre et la voix, Paris, Seuil, 1987, p. 312.

8. Ibid.

9. Ibid. p. 311. 
contours fixes ni d'essence transhistorique et pourtant elle est là, dans un état des discours sociaux bien différents du nôtre, avec les critères mêmes qui ont servi aux symbolistes pour distinguer la Religion de l'Art des intérêts de la "cohue démocratique".

Arrivés à ce point, peut-être trouverons-nous que le paradoxe est tel que nous pouvons vivre avec, nous installer confortablement en un lieu aporétique et voir venir. Nous ne serions pas encore tirés d'affaire.

La thèse selon laquelle les études littéraires ne peuvent définir positivement leur objet et travaillent sur des méthodologies et des concepts qui n'ont rien de propre à cet objet évanescent a été soutenue par d'autres que Todorov et Zumthor. Les conséquences que les uns et les autres en tirent varient cependant beaucoup. Le paradoxe ou la contradiction centrale est que les études dites littéraires, si elles doivent reconnaître n'avoir pas d'objet propre, peuvent s'enorgueillir d'avoir au contraire un ensemble extrêmement riche de problématiques, de schèmes d'analyse, de démarches exégétiques, cet ensemble présentant le grand avantage, ou le seul tort selon les points de vue, de ne servir guère à faire apparaître ce caractère de "textes ayant en soi leurs propres fins" qui, obscurément, est censé définir la particularité du fait littéraire.

À mesure même que, du formalisme au structuralisme et à notre époque enfin, parfois dite "post-structuraliste" avec le renouveau de l'herméneutique, le développement de l'analyse de la réception, de l'analyse intertextuelle, de la critique déconstructionniste, etc. - à mesure que les études littéraires ont développé des moyens d'analyse et de critique de plus en plus puissants et exigeants, loin des fades commentaires intuitifs et des ternes gloses philologiques de l'ancienne critique, dans cette mesure, l'objet "littérature» s'est dissipé, son illusoire évidence s'est dissoute. Les études littéraires ne se sont enrichies de paradigmes et d'instruments nouveaux, elles n'ont renoncé au 
subjectivisme brillant et au positivisme poussif que pour découvrir qu'à mesure de ce développement, l'autonomie et la spécificité des belles lettres étaient irrévocablement questionnées et démantelées. Ferdinand Brunetière et Jules Lemaître croyaient à l'éclatante évidence du fait littéraire. Gustave Lanson croyait pouvoir se démarquer d'eux en fondant une méthode historique positiviste qui ne partait pas moins du fait littéraire comme d'une donnée empirique sans problème. Mais dès lors que les formalistes russes se sont mis à la tâche de fonder une "science de la littérature", c'est la spécificité de cette science, l'autonomie de la "série littéraire" (Tynianov) par rapport à la topologie globale du système culturel, l'adéquation des méthodes développées par rapport à leur objet prétendu qui se sont mises à faire problème ${ }^{10}$. Sans doute, la prétention résurgente de substituer aux études sectorielles une sémiotique générale qui englobât l'ensemble de la culture objectivée en ses artefacts, cette prétention ou cet espoir n'ont jamais abouti, si aboutir veut dire convertir un grand nombre de chercheurs à adopter un paradigme fondamental et une sorte de programme commun de recherches. De Mukarovsky à Eco et à Greimas, les grands systèmes sémiotiques ont été ou bien négligés, largement ignorés (ce qui demeure le cas pour Mukarovsky), ou bien sont devenus la doctrine groupusculaire de disciples fervents pour lesquels la complexité du monde sert plutôt à justifier la doctrine que le modèle doctrinal ne sert pas à rendre raison du monde. L'éclectisme flou qui tient lieu de contrat de bonne entente dans les études littéraires fait que ces constructions théoriques sont là, comme les pavillons abandonnés d'une exposition universelle après sa ferme ture, ouvertes au badaud, au milieu des gravats et les

10. Jurii Tynianov, “O literaturnoj evoljucii», trad. “Über die literarische Evolution " dans J. Striedter, Texte der Russischen Formalisten, I., Munich, Fink, 1969, p. 433-62. 
portes battantes. De sorte que les études littéraires ont des méthodes mais pas d'objet, tandis que la sémiotique culturelle générale qui nous semble sans doute avoir un objet n'a qu'une abondance de constructions systématiques qui semblent inaptes à rallier l'ensemble des chercheurs où à alimenter les recherches.

On parle depuis plus de vingt ans en GrandeBretagne de la "crise des études littéraires" et peut-être les Britanniques ont-ils été les premiers à regarder cette crise en face et à chercher à en tirer des conséquences logiques ${ }^{11}$. Crise du concept de littérature, crise institutionnelle de l'enseignement des lettres dans le système scolaire : ces deux crises n'appartiennent pas à la même série, mais elles sont concomitantes. La "culture du pauvre" planétaire qu'instaurent les holdings internationaux de l'industrie culturelle aurait pu servir d'alibi à un repli élitiste. Un grand nombre d'Anglais ont choisi au contraire de délaisser les études littéraires pour des "cultural studies" et pour un enseignement non dépourvu de volontarisme militant ne prétendant s'intéresser qu'à ce qui dans la production écrite ouvrière, féminine, tiers-mondiste et marginale semblait résister à l'emprise hégémonique des idées reçues et des intérêts qui s'attachent à la culture "désintéressée». Nous savons que Terry Eagleton dans son Literary Theory conclut au rejet de la chose ainsi nommée «littérature» comme objet d'étude privilégié :

L'axiome selon lequel ce que l'on nomme présentement «littérature" sera toujours et partout le centre d'attention le plus important ne doit pas être posé comme un a priori. Un tel dogmatisme n'a pas de place dans le champ des études culturelles ${ }^{12}$.

11. Voir Robert Young, “Une tradition en crisew dans Études francaises, 23 : 1-2, 1987-88, p. 47-74.

12. Terry Eagleton, Literary Theory, Londres, Blackwell, 1983, p. 213. (Ma traduction). 
II précisait, pour que ses conclusions n'apparaissent pas comme une vaine déclaration de principe :

Cela signifierait que les départements de littérature tels que nous les connaissons présentement dans l'enseignement supérieur cesseraient d'exister.

J'avoue adhérer aux thèses de Terry Eagleton sans adhérer du tout au volontarisme quasi-militant ambigu auquel il aboutit. Je me borne ici à les discuter, de façon essentiellement technique, par trois propositions complémentaires :

Les études littéraires n'ont pas d'objet stable et délimité. L'argument du texte autotélique, non référentiel, repris encore par Paul Zumthor, ne tient pas : toutes les analyses qui donnent pour objet à la littérature le utravail de la forme" aboutissent à faire apparaître que ce travail est un travail sur le discours social qui, loin de conduire à l'évanescence sémantique, est un accélérateur exégétique et un "orchestrateur" des significations latentes inscrites dans le discours social; la perte d'une fonction doxique unidimensionnelle attachée aux mots et aux locutions reconnaissables dans le texte ne constitue aucunement une perte de référence, de fonction référentielle mais donne à la référentialité une définition positiviste étroite.

J'ai fait allusion plus haut à l'exemple de "poétisation" d'un fait divers à quoi se livre Gérard Genette par la simple altération d'une disposition typographique en vers libres :

\author{
Hier sur la Nationale sept \\ Une automobile \\ Roulant à cent à l'heure s'est jetée \\ Sur un platane \\ Ses quatre occupants ont été \\ Tués $^{13}$.
}

13. Voir note 4. 
S'il est vrai que, ainsi monté en épingle et décontextualisé, le bref «flash" d'agence prend une dimension herméneutique et quasiment métaphysique (on y lira la banalisation moderne du tragique, dit Jonathan Culler ${ }^{14}$ ), il est faux cependant que l'avatar "poétique" du fait divers prive le texte de toute référence et enferme le ureadymade" poétique dans sa propre immanence formelle. Au contraire, toute interprétation du ready-made le fera apparaître comme un travail sur le discours social par une distanciation déconstructive qui défamiliarise un sociolecte journalistique pour faire dire l'absurde, l'anonymat, pour accroître donc la richesse de la signification et intensifier le pathétique et non pour couper le texte du flux sociodiscursif.

Deuxième proposition que j'ai déjà développée : le marché des méthodologies dites "littéraires" est de peu de secours pour disserter de la spécificité, toujours alléguée, du fait littéraire, en ceci que cette spécificité, historiquement variable et multiple, est fonction de l'économie globale du discours social et ne se comprend pas en termes immanentistes, mais en termes de travail interdiscursif. Complémentairement, les méthodologies littéraires sont des méthodologies sociodiscursives perverties par leur application à un objet fétichisé.

Impossible aujourd'hui de construire une narratologie en ne se référant qu'aux formes littéraires du récit, de même qu'il est impossible de produire une théorie de la métaphore en limitant ses analyses au domaine des belles lettres. II faut pour parler métaphores, parler argot, échange oral, catachrèses, néologies, jargons scientifiques et typologie des discours sociaux...

Troisième thèse qui complète les deux premières : le seul objet d'étude qui, dans son autonomie relative en cul-

14. Jonathan Culler, "La littérarité», dans Marc Angenot et al. Théorie littéraire, Paris, P.U.F., 1989, p. 40. 
ture, forme une entité propre et un champ global d'interaction, c'est le discours social tout entier dans la complexité de sa topologie, de sa division du travail, et c'est dans le cadre d'une analyse et d'une théorie du discours social qu'on pourra isoler certains écrits, appartenant parfois au champ littéraire, dont le travail critique sur l'intertexte apparaîtra comme révélateur, intéressant, innovateur, significatif, pour des motifs contingents à l'ordre global des discours, aux effets d'occultation et de cloisonnement que révèlent contradictoirement les anaphores, les discordances, les paradoxismes inscrits dans le texte examiné. C'est au discours social, dans la complexité cacophonique de ses langages, de ses schémas cognitifs, de ses migrations thématiques que s'appliquent les méthodologies des études littéraires - «débarrassées" de ce qu'elles ont de fétichiste et de formaliste - et ce n'est que dans le discours social que peuvent se réconcilier, avec un certain degré d'objectivation et de démonstrabilité, les trois étapes traditionnelles de la description, de l'interprétation et de l'évaluation des textes, des œuvres, et des genres et discours qui coexistent et interfèrent dans une culture donnée. Le texte littéraire inscrit du discours social et le travaille. Mais le texte littéraire demeure une pure entéléchie : le travail sur les discours sociaux n'est pas une tâche transhistorique qui va de soi, ce travail est toujours problématique et ses stratégies sont multiples, contraintes et, dans une même société, divergentes par leurs moyens et leurs fonctions. Le discours social apparaît comme un dispositif problématologique, fait de leurres, d'énigmes, de dilemmes et de questionnements. Si les textes, littéraires ou non, se réfèrent au réel, cette référence s'opère dans la médiation des langages et des discours qui, dans une société donnée "connaissent" différentiellement et même de façon antagoniste, le réel dont je ne puis rien dire antérieurement aux diverses manières dont il est connu ${ }^{15}$. Si mes thèses sont en accord relatif avec les perspectives heuristiques des 
chercheurs dits littéraires - je pense ici au regretté Raymond Williams, à Edmond Cros, Claude Duchet, Charles Grivel et plusieurs amis et collègues au Québec - je poserais plus radicalement cependant l'ordre heuristique qui en découle. Sans une théorie et une pratique d'analyse du discours social, lequel est bien plus et bien autre chose que l'intuition qu'on en a, il n'est guère possible d'aborder le corpus littéraire tout de go, sans tomber dans l'a priori, l'intuition incontrôlée, l'imputation aux caractères formels de l'objet des fonctions interdiscursives du texte. Ce qui manque aujourd'hui - au-delà des constructions élitistes de l'histoire des idées et des interprétations mécanistes de la critique dite "idéologique" - c'est une théorie et une histoire du discours social, et la tâche que je me donne est d'y contribuer et de renoncer par là-même aux blandices du "texte pur", concevant la tâche du doxographe, dirons-nous, du critique socio-discursif, comme de caractère fondamentalement historiographique et sociologique.

Marc Angenot Université McGill

15. Voir Régine Robin et Marc Angenot, «L'inscription du discours social dans le texte littéraire», Sociocriticism, Pittsburgh, no 1, juillet 1985, p. 53-82. 